\title{
A Mathematical Model and Analysis of an SVEIR Model for Streptococcus Pneumonia with Saturated Incidence Force of Infection
}

\section{Opara Chiekezi Zephaniah, Uche-Iwe Ruth Nwaugonma, Inyama Simeon Chioma, Omame Adrew}

Department of Mathematics, Federal University of Technology, Owerri, Nigeria

\section{Email address:}

Zephc.opara@yahoo.com (Opara C. Z.), ucheruthy@yahoo.com (Uche-Iwe R. N.), scinyama2011@yahoo.com (Inyama S. C.), omame2020@gmail.com (Omame A.)

\section{To cite this article:}

Opara Chiekezi Zephaniah, Uche-Iwe Ruth Nwaugonma, Inyama Simeon Chioma, Omame Adrew. A Mathematical Model and Analysis of an SVEIR Model for Streptococcus Pneumonia with Saturated Incidence Force of Infection. Mathematical Modelling and Applications. Vol. 5, No. 1, 2020, pp. 16-38. doi: 10.11648/j.mma.20200501.13

Received: October 16, 2019; Accepted: November 28, 2019; Published: February 19, 2020

\begin{abstract}
In this paper, the dynamics of SVEIR model with saturated incidence force of infection and saturated vaccination function for Streptococcus pneumonia (that is, model that monitors the temporal transmission dynamics of the disease in the presence of preventive vaccine) was formulated and analyzed. The basic reproduction number that determines disease extinction and disease survival was revealed. The existing threshold conditions of all kinds of the equilibrium points are obtained and proved to be locally asymptotic stable for disease-free equilibrium using linearization method and Lyapunov functional method for Endemic equilibrium. Qualitative Analysis of the model was obtained and the positive of solution obtained. It was revealed that the model is positively -invariant and attracting. Thus the region is positively invariant. Hence, it is sufficient to consider the dynamics of the model (1) in the given region. In this region, the model can be considered as been epidemiologically and mathematically well-posed. The governing model was normalized and also Adomian Decomposition method was used to compute an approximate solution of the nonlinear system of differential equations governing the model. Maple was used in carrying out the simulations (numerical solutions) of the model. Graphical results were presented and discussed to illustrate the solution of the problem. The achieved results reveal that the disease will die out within the community if the vaccination coverage is above the critical vaccination proportion. The study indicates that we should improve the efficiency and enlarge the capacity of the treatment to control the spread of disease.
\end{abstract}

Keywords: Mathematical Model, SVEIR Model, Streptococcus Pneumonia, Saturated Incidence Force Ofinfection

\section{Introduction}

Streptococcus pneumonia is a facultative anaerobic bacterium, gram-positive which have the shape of a lancet. It exists in more than 90 serotypes. Most of these serotypes can originate from different diseases, with little of serotypes being the major factor of pneumococcal infections [6]. Research showed that this gram-positive bacterium "Pneumococcus "inhabits the respiratory tract and perhaps can be secluded from the nasal part of the pharynx, lying behind the nose of about 5-90\% among individuals who are healthy, relatively on the population and setting. Adults forms about $5-10 \%$ carriers while 20 $60 \%$ of school-aged children are probably carriers.
Investigations also revealed that about $50-60 \%$ of service personnel may be carriers. The time frame of carriage differs among different age group as it tends to be longer in children than adults. Furthermore, it is still unclear for researchers on how carriage and individuals' ability to develop natural immunity are related.

Most of the serious infections that are communityacquired (like; meningitis and bacteremia) has been traced to Streptococcus pneumonia being one of the primary cause among children who are below the age of 5 years, $[20,36]$. Among patients who are HIV positive, Sphas also be linked to be the major cause of sepsis and bacteremia, [22]. The infections instigated by $S$. pneumonia are one of the principal causes of death among 
children in Nigeria today, [13]. This is recorded due to the poor access to adequate healthcare and can also be attributed to high endemicity of HIV infect, [4]. Studies have associated children who are colonized with S. pneumonia to have higher chance of being hospitalized compared those who are not colonized, [12].

A large number (especially young children) of people are still battling with infections caused by these bacteria, the elderly or patients with low immunity irrespective of the efforts to ensure that rate of mortality and morbidity among children in developing countries is reduced, [5]. Research have revealed that $\mathrm{S}$. pneumonia is the most popular bacteria isolated from blood and sputum samples of children with severe S. pneumonia, [33]. Pneumococcal disease is generally preceded by asymptomatic colonization, which is mainly high in children, [5, 22]. Depending on the considered case, invasive pneumococcal diseases occurs immediately after colonization, and it has been revealed that the streptococcal nasopharyngeal carriage prevalence in unvaccinated children is similarly high in Africa ranging from 7-90\%, [3, 4, 9, 21, 22].

The threat of $S p$ infection has been increasing despite interventions by widely available antibiotics, due to the increasing presence of multiple antibiotic-resistant $S p$ strains, $[35,31]$.

There is an urgent call for a better informed intervention targeted in mitigating the early occurrence of $\mathrm{S} p$-mediated pathology through vaccination and treatment with little antibiotics [37, 38]. Formerly, proposed mathematical models considered $S p$ infections in the lung which is a normally sterile site of the airway epithelium. There have been numerous epidemic models designed and explored, also many vaccination campaigns to prevent eradicate or mitigate the speed transmission of the infectious childhood diseases (for example measles, tuberculosis, and flu). Bilinear incidence rate $\beta S I$ has been frequently applied in many epidemic models according to $[1,2,30,39,40,41]$. The saturated incidence rate $\beta S I(1+\alpha \mathrm{I})$ was introduced by [11]. This reveals that if $\beta \mathrm{I}$ (which estimates the infection force at time of disease total invasion in the susceptible population) is large together with $1 /(1+\alpha \mathrm{I})$ (which estimates the reacting effect out of the behavioral change of the susceptible population at the time we have a crowding effect of the infected population), then the model is certainly to be saturated. It comes up with the concept of continuous treatment in an SIR model as follows:

$$
h(I)= \begin{cases}r, & I>1 \\ 0, & I<1\end{cases}
$$

$$
h(I)= \begin{cases}r, & I>1 \\ 0, & I<1\end{cases}
$$

That takes care of some fraction of individuals who are successfully treated and the rest unsuccessful, [43]. Further, Wang considered the following piecewise linear treatment function

$$
h(I)=\left\{\begin{array}{l}
r I, 0 \leq I \leq 1 \\
y I_{0}, I>I_{0}
\end{array}\right.
$$

We agree this to be more reasonable than the usual linear function. This revealed that if treatment is delayed for infected individuals, the efficiency will be drastically affected. Furthermore, the continuous and differentiable saturated treatment function was introduced and used given as $h(I)=r I /(1+k I)$, where $\mathrm{r}>0, \mathrm{k}>0, r$ implies rate of cure, and $\mathrm{k}$ estimates the treatment delay level of the infected individuals. This reveals that if Iis small, then the treatment function tends to $r \mathrm{I}$, while it tends to $r / k$ if $I$ is large, [10, 45].

In the maximum amount because the dynamics of SIR or SIS epidemic models with the saturated incidence rate are recurrently utilized in several literatures. We have a tendency to still have very little researches regarding the saturated treatment operate even within the SEIR epidemic models. Other works which we looked at in this work are: $[8,15,18$, $28,34,25,29,45,46,47]$.

\section{Model Formulation}

\subsection{Assumptions of the Model}

The assumptions of the model are stated below:

1. We assume that the saturated incidence force of infection rate is $\frac{\beta I}{1+\alpha I}$

2. People in the Vaccinated compartment can be infected.

3. People can be infected only through contacts with infectious people except those who are immune.

4. Recovered individuals may go back to the susceptible class.

5. The population is homogeneously mixed (A population that interacts with one another to the same degree andfixed).

\subsection{Symbols and Parameter of the Model}

Below are the symbols and parameters of the proposed model.

Table 1. Symboles and parameters.

\begin{tabular}{ll}
\hline Symbols and Parameters & Description \\
\hline $\mathrm{S}$ & Susceptible Individuals at time $\mathrm{t}$ \\
$\mathrm{V}$ & Vaccinated Individuals at time $\mathrm{t}$ \\
$\mathrm{E}$ & Exposed individuals at time $\mathrm{t}$ \\
$\mathrm{I}$ & Infectious individuals at time $\mathrm{t}$ \\
$\mathrm{R}$ & Recovered individuals at time $\mathrm{t}$ \\
$\mu(\mathrm{Mu})$ & Per capita natural mortality rate (Natural death rate) \\
$\beta($ Beta $)$ & Contact rate \\
\hline
\end{tabular}




\begin{tabular}{ll}
\hline Symbols and Parameters & Description \\
\hline$\Re_{0}$ & Basic reproduction number \\
$\xi(\mathrm{Xi})$ & Effectiveness of vaccine \\
$(1-\xi)$ & Rate at which vaccines get waned \\
$\mathrm{N}$ & Total population \\
$\mathrm{P}$ & Fraction of recruited individuals into the vaccinated population \\
$\sigma(\mathrm{Sigma})$ & infectious rate of exposed individuals \\
$\gamma \quad$ Gama & Recovery rate of infected individuals \\
$\omega($ Omega $)$ & Susceptible rate of vaccinated individuals \\
$\Lambda($ Capital Lambda) & Recruitment rate into the susceptible compartment \\
$\lambda=\frac{\beta I}{1+\alpha I}$ & Saturated incidence force of infection \\
$\rho(\mathrm{Rho})$ & Per capita rate of recovered individuals going back to the Susceptible class. \\
$\mathrm{A}$ & Death as a result of the infection \\
$\mathrm{F}_{\mathrm{i}}$ & The rate of appearance of new infection in compartment I \\
$v_{i}^{+}$ & The rate of transfer of individuals into the infected compartment \\
$v_{i}^{-}$ & The transfer of individual out of the infected compartment \\
\hline
\end{tabular}

Now we have

$$
N(t)=S(t)+V(t)+E(t)+I(t)+R(t)
$$

\subsection{Flow Diagram of the Model}

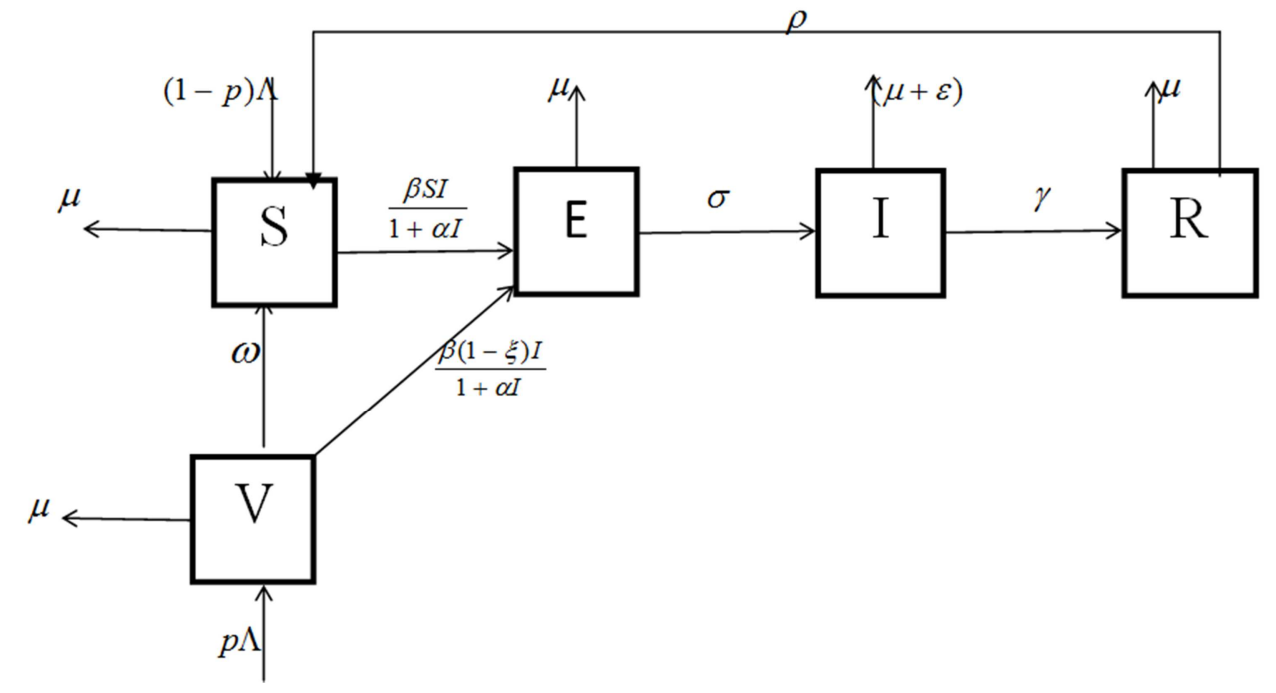

Figure 1. Schematic Diagram of the Model.

\subsection{The Model Equation}

Applying the symbols and parameters, assumptions and flow diagram, we now formulate the model equations as follows:

$$
\left.\begin{array}{l}
\frac{d S}{d t}=(1-p) \Lambda+\omega \Lambda+\rho R-\frac{\beta I}{1+\alpha I} S-\mu S \\
\frac{d V}{d t}=p \Lambda-\frac{\beta(1-\xi) V I}{1+\alpha I}-(\mu+\omega) V \\
\frac{d E}{d t}=\frac{\beta I}{1+\alpha I} S+\frac{\beta(1-\xi) V I}{1+\alpha I}-(\mu+\sigma) E \\
\frac{d I}{d t}=\sigma E-(\mu+\varepsilon+\gamma) I \\
\frac{d R}{d t}=\gamma I-(\sigma+\rho) R
\end{array}\right\}
$$


Where $\lambda=\frac{\beta I}{1+\alpha I}$ is the saturated incidence force of infection.

\section{Qualitative Analysis}

\subsection{Positivity of Solutions}

We prove the positivity of the solution by stating and proving the Theorem 3.1 below.

Theorem 3.1:

Suppose we have the initial solution of our model to be $\{S(0), V(0), E(0), I(0), R(0) \geq 0\} \in \varphi$. Then for all $t \geq 0$ the solution set $\{S(t), V(t), E(t), I(t), R(t)\}$ the system (3) remains positive

Proof:

From the first equation of (3) we have

$$
\begin{gathered}
\frac{d S}{d t} \geq-\mu S \Rightarrow \frac{d S}{S} \geq-\mu d t \\
\Rightarrow \ln S \geq-\mu t+c \Rightarrow S \geq e^{-\mu t+c}=S_{0} e^{-\mu t} \geq 0 \text { where } S_{0}=e^{c}
\end{gathered}
$$

From the second equation of (3) we have

$$
\begin{gathered}
\frac{d V}{d t} \geq-(\mu+\omega) V \\
\Rightarrow \frac{d V}{V} \geq(\mu+\omega) d t \Rightarrow \ln V \geq(\mu+\omega) t+c \Rightarrow V \geq e^{(\mu+\omega) t+c}=V_{0} e^{(\mu+\omega) t} \text { where } V_{0}=e^{c}
\end{gathered}
$$

From the third equation of (3) we have

$$
\begin{gathered}
\frac{d E}{d t} \geq-(\mu+\sigma) E \Rightarrow \frac{d E}{E} \geq-(\mu+\sigma) d t \\
\Rightarrow \ln E \geq-(\mu+\sigma) t+c \Rightarrow E \geq e^{-(\mu+\sigma) t+c}=E_{0} e^{-(\mu+\sigma)} \text { where } E_{0}=e^{c}
\end{gathered}
$$

From the fourth equation of (3) we have

$$
\begin{gathered}
\frac{d I}{d t} \geq-(\mu+\varepsilon+\gamma) I \Rightarrow \frac{d I}{I} \geq-(\mu+\varepsilon+\gamma) d t \\
\Rightarrow \ln I \geq-(\mu+\varepsilon+\gamma) t+c \Rightarrow I \geq e^{-(\mu+\varepsilon+\gamma) t+c}=I_{0} e^{-(\mu+\varepsilon+\gamma) t} \text { where } I_{0}=e^{c}
\end{gathered}
$$

From the fifth equation of (3) we have

$$
\begin{gathered}
\frac{d R}{d t} \geq-(\mu+\rho) R \Rightarrow \frac{d R}{R} \geq-(\mu+\rho) d t \\
\ln R \geq-(\mu+\rho) t+c \Rightarrow R \geq e^{-(\mu+\rho) t+c}=R_{0} e^{-(\mu+\rho)} \text { where } R_{0}=e^{c}
\end{gathered}
$$

Hence the solution set $\{S(t), V(t), E(t), I(t), R(t)\}$ of the system (3) is positive for all $t \geq 0$.

Remark 1: $e^{k}>0$ for all real values of $\mathrm{k}$.

Theorem 3.2 (Invariant Region)

The following feasible region of the model (3)

$$
\Omega=\left\{(S, V, E, I, R) \in \mathrm{R}_{+}^{5}: S+V+E+I+R \leq \frac{\Lambda}{\mu}\right\}
$$


is a positive invariant region and attracting.

Proof: From model (3),

$$
\begin{gathered}
S+V+E+I+R=N \Rightarrow \frac{d N}{d t}=\frac{d S}{d t}+\frac{d V}{d t}+\frac{d E}{d t}+\frac{d I}{d t}+\frac{d R}{d t} \\
\frac{d N}{d t}=\Lambda-\mu S-\mu V-\mu E-\mu I-\mu R=\Lambda-\mu(S+V+E+I+R)=\Lambda-\mu N \\
\therefore \frac{d N}{d t}=\Lambda-\mu N \\
\Rightarrow \frac{d N}{d t}=\Lambda-\mu N \Rightarrow \frac{d N}{d t}+\mu N=\Lambda \Rightarrow \frac{d}{d t}(\mu N)=\Lambda+c e^{-\mu} \Rightarrow N=\frac{\Lambda}{\mu}+\frac{c e^{-\mu}}{\mu} \Rightarrow N \leq \frac{\Lambda}{\mu} \geq 0 \\
\therefore \Omega=\left\{(S, V, E, I, R) \in \mathfrak{R}_{+}^{5}: S+V+E+I+R \leq \frac{\Lambda}{\mu}\right\}
\end{gathered}
$$

is positive invariant and attracting.

Thus $\Omega$ is positive invariant. Therefore, it is very significant to study the behavior of our model (4) in region $\Omega$, because in this region, the model is epidemiologically and mathematically meaningful, [14].

\subsection{Existence of Steady States}

The existence of steady state of the model occurs at

$$
\begin{gathered}
\frac{d S}{d t}=\frac{d V}{d t}=\frac{d E}{d t}=\frac{d I}{d t}=\frac{d R}{d t}=0 \\
(1-p) \Lambda+\omega \Lambda+\rho R-\frac{\beta I}{1+\alpha I} S-\mu S=0 \\
p \Lambda-\frac{\beta(1-\xi) V I}{1+\alpha I}-(\mu+\omega) V=0 \\
\frac{\beta I}{1+\alpha I} S+\frac{\beta(1-\xi) V I}{1+\alpha I}-(\mu+\sigma) E=0 \\
\sigma E-(\mu+\varepsilon+\gamma) I=0 \\
\gamma I-(\sigma+\rho) R=0
\end{gathered}
$$

Solving (7)-(11) we have

From (7),

$$
(1-p) \Lambda+\omega \Lambda+\rho R-\frac{\beta I}{1+\alpha I} S-\mu S \Rightarrow S^{*}=\frac{(1-p) \Lambda+\omega V^{*}+\rho R^{*}}{\left(\lambda \beta I^{*}+\mu\right)}
$$

From (8)

$$
\begin{gathered}
p \Lambda-\frac{\beta(1-\xi) V I}{1+\alpha I}-(\mu+\omega) V=0 \Rightarrow p \Lambda(1+\alpha I)=\beta(1-\xi) V I-(\mu+\omega)(1+\alpha I) V \\
\Rightarrow V^{*}=\frac{p \Lambda}{\lambda \beta(1-\xi) I^{*}-(\mu+\omega)}
\end{gathered}
$$

From (9) 


$$
\frac{\beta I}{1+\alpha I} S+\frac{\beta(1-\xi) V I}{1+\alpha I}-(\mu+\sigma) E=0 \Rightarrow E^{*}=\frac{\lambda \beta I^{*}\left[S^{*}+(1-\xi) V^{*}\right]}{(\mu+\sigma)}
$$

From (10)

$$
\sigma E-(\mu+\varepsilon+\gamma) I=0 \Rightarrow I^{*}=\frac{\sigma E^{*}}{(\mu+\varepsilon+\gamma)}
$$

From (11)

$$
\gamma I-(\sigma+\rho) R=0 \Rightarrow R^{*}=\frac{\gamma I^{*}}{(\mu+\rho)}
$$

Summarizing we have that the disease-free steady state is

$$
\xi^{0}=\left(S^{*}, 0,0,0,0\right)
$$

and the endemic steady states is

$$
\xi_{E}=\left(S^{*}, V^{*}, E^{*}, I^{*}, R^{*}\right)
$$

where $S^{*}, V^{*}, E^{*}, I^{*}, R^{*}$ are as defined below.

$$
\left.\begin{array}{l}
S^{*}=\frac{(1-p) \Lambda+\omega V^{*}+\rho R^{*}}{\left(\lambda \beta I^{*}+\mu\right)} \\
V^{*}=\frac{p \Lambda}{\lambda \beta(1-\xi) I^{*}-(\mu+\omega)} \\
E^{*}=\frac{\lambda \beta I^{*}\left[S^{*}+(1-\xi) V^{*}\right]}{(\mu+\sigma)} \\
I^{*}=\frac{\sigma E^{*}}{(\mu+\varepsilon+\gamma)} \\
R^{*}=\frac{\gamma I^{*}}{(\mu+\rho)}
\end{array}\right\}
$$

\subsection{Disease Free Equilibrium (DFE) of the Model}

The model system (1) has a steady state at a given period where there is no S. Pneumonia in the population under consideration. That is; when $E^{0}=I^{0}=0$. Hence, the disease-free equilibrium (DFE) denoted as $\xi^{0}$, of the model system (1) is obtained by Substituting $E^{0}=I^{0}=0$ into (18)

Hence the disease free equilibrium of system (1) of the model equation is given by

$$
\xi^{0}=\left(S^{0}, V^{0}, E^{0}, I^{0}, R^{0}\right)=\left(\frac{\Lambda \omega+\Lambda \mu(1-p)}{\mu(\mu+\omega)}, \frac{\Lambda p}{\mu+\omega}, 0,0,0\right)
$$

\subsection{The Model Basic Reproduction Number}

The local stability is established by using the next generation operator method on the system.

The basic reproduction number $R_{0}$ is defined as the effective number of secondary infections caused by an infected individual during his/her entire period of infectiousness, [42].

When $R_{0}<0$, it implies that each individual produces on average less than one new infected individual and hence the disease dies out with time. On the other hand, when $R_{0}>0$, it means each individual produces more than one new infected individual and hence the disease is able to invade the susceptible population. However, $R_{0}=0$ is the threshold below which the generation of secondary cases is insufficient to maintain the infection within human community? The basic reproduction number cannot be determined from the structure of the mathematical model alone, but depends on the definition of infected and uninfected compartments.

This definition is given for the models that represent 
spread of infection in a population. It is obtained by taking the largest (dominant) eigenvalue or spectral radius of

$$
\rho\left(F_{i} V^{-1}\right)
$$

where

$$
F_{i}=\left[\frac{\partial f_{i}\left(\xi^{0}\right)}{\partial X_{j}}\right] \text { and } V_{i}=\left[\frac{\partial v_{i}\left(\xi^{0}\right)}{\partial X_{j}}\right]
$$

$f_{i}$ is the rate of appearance of new infection in compartment $i$ and $v_{i}=v_{i}^{-}-v_{i}^{+}$

$v_{i}{ }^{-}$is the transfer of individuals out of the compartment $i$,

$v_{i}{ }^{+}$is the rate of transfer into compartment $\mathrm{i}$ by any other means,

$\xi^{0}$ is the disease free equilibrium

Then by linearizing $f_{i}$ and $v_{i}$, and evaluating at the disease free equilibrium point we obtain the associated matrices $F_{i}$ and $V_{i}$ respectively.

The infected compartments are, $E$ and $I$ hence a

$$
\begin{gathered}
V^{-1}\left(\xi^{0}\right)=\frac{1}{(\sigma+\mu)(\mu+\varepsilon+\gamma)}\left(\begin{array}{cc}
\mu+\varepsilon+\gamma & 0 \\
\sigma & \sigma+\mu
\end{array}\right) \\
F V^{-1}=\frac{1}{(\sigma+\mu)(\mu+\varepsilon+\gamma)}\left(\begin{array}{cc}
0 & \beta\left(S^{0}+(1-\xi) V^{0}\right) \\
0 & 0
\end{array}\right)\left(\begin{array}{cc}
\mu+\varepsilon+\gamma & 0 \\
\sigma & \sigma+\mu
\end{array}\right)=\left(\begin{array}{cc}
\frac{\sigma \beta\left(S^{0}+(1-\xi) V^{0}\right)}{(\sigma+\mu)(\mu+\varepsilon+\gamma)} & \frac{\beta\left(S^{0}+(1-\xi) V^{0}\right)}{\mu+\varepsilon+\gamma} \\
0 & 0
\end{array}\right)
\end{gathered}
$$

The basic reproduction number is given by $R_{0}=\rho\left(F V^{-1}\right)$, that is, the highest eigenvalue of $F V^{-1}$ is Hence

$$
R_{0}=\frac{\sigma \beta \Lambda[\mu(1-p \xi)+\omega]}{\mu(\mu+\omega)(\sigma+\mu)(\mu+\varepsilon+\gamma)}
$$

\subsection{Local Stability of the Disease-free Steady State}

Theorem 3.3: The disease free equilibrium $\left(\xi^{0}\right)$ is locally asymptotically stable if $R_{0}<1$ and unstable if $R_{0}>1$ Proof:

We prove the locally asymptotically stability of the disease free equilibrium $\left(E^{0}\right)$ of model (1) using linearization approach. We linearize the model equation to obtain the Jacobian matrix:

$$
J=\left(\begin{array}{ccccc}
(\lambda \beta+\mu) & \omega & 0 & -\lambda \beta S & \rho \\
0 & -\lambda \beta(1-\xi) I(\mu+\omega) & 0 & \lambda \beta(1-\xi) V & 0 \\
\lambda \beta I & \lambda \beta(1-\xi) I & -(\mu+\sigma) & \lambda \beta S+\lambda \beta(1-\xi) V & 0 \\
0 & 0 & \sigma & -(\mu+\varepsilon+\gamma) & 0 \\
0 & 0 & 0 & \gamma & -(\mu+\rho)
\end{array}\right)
$$

At the DFE J becomes; 


$$
J=\left(\begin{array}{ccccc}
(1-\rho) & \omega & 0 & -\lambda \beta S & \rho \\
0 & -\lambda \beta(1-\xi) I(\mu+\omega) & 0 & \lambda \beta(1-\xi) V & 0 \\
\lambda \beta I & \lambda \beta(1-\xi) I & -(\mu+\sigma) & \lambda \beta S+\lambda \beta(1-\xi) V & 0 \\
0 & 0 & \sigma & -(\mu+\varepsilon+\gamma) & 0 \\
0 & 0 & 0 & \gamma & -(\mu+\rho)
\end{array}\right)
$$

Hence the characteristics equation of the above Jacobian is

$$
|J-\lambda I|=\left[\begin{array}{ccccc}
\lambda+k_{1} & \omega & 0 & \frac{-\beta}{\mu} k_{1} & -\rho \\
0 & -(\mu+\omega)-\lambda & 0 & \beta k_{1} & 0 \\
0 & 0 & -(\mu+\sigma)-\lambda & \frac{-\beta}{\mu}\left(k_{1}+\mu k_{1}\right) & 0 \\
0 & 0 & \sigma & \lambda+(\mu+\varepsilon+\gamma) & 0 \\
0 & 0 & 0 & \gamma & -(\mu+\rho)-\lambda
\end{array}\right]=0
$$

where $I$ is $5 \times 5$ identity matrix

That implies that

$$
\begin{aligned}
& k_{1}=\frac{\Lambda \omega+\Lambda \mu(1-\rho)}{(\mu+\omega)}, k_{2}=\frac{(1-\xi) \Lambda p}{(\mu+\omega)} \\
& \text { where }\left(k_{1}-\lambda\right)[(\mu+\omega)-\lambda][(\mu+\rho)-\lambda]\left[\lambda^{2}+(2 \mu+\sigma+\xi+\gamma) \lambda+(\mu+\sigma)(\mu+\xi+\gamma)-\frac{\alpha \beta}{\mu}\left(k_{1}+\mu k_{2}\right)\right]=0
\end{aligned}
$$

Obviously,

$$
\lambda_{1}=k_{1}, \lambda_{2}=-(\mu+\omega), \lambda_{3}=-(\mu+\rho) \text { and } \lambda^{2}+(2 \mu+\sigma+\xi+\gamma) \lambda+\left[(\mu+\sigma)(\mu+\xi+\gamma)-\frac{\alpha \beta}{\mu}\left(k_{1}+\mu k_{2}\right)=0\right]
$$

Letting $A_{2}=1, A_{1}=2 \mu+\sigma+\xi+\gamma$ and $A_{0}=(\mu+\sigma)(\mu+\xi+\gamma)-\frac{\alpha \beta}{\mu}\left(k_{1}+\mu k_{2}\right)$, we obtain the equation

$$
A_{2} \lambda^{2}+A_{1} \lambda+A_{0}=0
$$

Using the Routh-Hurwitz stability criterion according to [32]which state that all the roots of the characteristics equation (19) above have negative real parts if and only if the co-efficient $A_{i}$ are positive and matrices $H_{i}>0$ for $i=0,1,2$

Hence from (18) we see that

$$
A_{2}=1>0 ; A_{1}=2 \mu+\sigma+\xi+\gamma>0
$$

And

$$
\begin{aligned}
& A_{0}=(\mu+\sigma)(\mu+\xi+\gamma)-\frac{\sigma \beta[\wedge \omega+\lambda \mu(1-\xi \rho)]}{\mu(\mu+\omega)} \\
& \Rightarrow A_{0}=\mu(\mu+\omega)(\mu+\sigma)(\mu+\xi+\gamma)-\sigma \beta \wedge \omega-\sigma \beta \wedge \mu+\sigma \beta \rho \xi \\
&=\mu(\mu+\omega)(\mu+\sigma)(\mu+\xi+\gamma)+\sigma \beta \rho \xi-\sigma \beta \wedge(\omega+\mu) \\
&=(\mu+\omega)[\mu(\mu+\sigma)(\mu+\xi+\gamma)]+\sigma \beta \rho \xi>0
\end{aligned}
$$

Again, the Hurwitz Matrix

$$
H=\left(\begin{array}{cc}
A_{1} & 0 \\
A_{0} & A_{2}
\end{array}\right)
$$


With

$$
\begin{aligned}
& H_{1}=\left|A_{1}\right|=A_{1}>0 \\
& H_{2}=\left(\begin{array}{ll}
A_{1} & 0 \\
A_{0} & A_{2}
\end{array}\right)=A_{1} A_{2}>0
\end{aligned}
$$

This therefore shows that all the eigenvalues of the Jacobian of the system have negative real parts. Hence, the DFE is locally asymptotically stable.

\subsection{Global Stability of Disease-free Steady State}

Theorem 3.4: If $R_{0} \leq 1$ for the model (4) then the infection-free equilibrium state $\left(\xi^{0}\right)$ is the only non-negative equilibrium state of the model (4) and it is globally asymptotically stable.

Proof:

Let $L=c_{1} E+c_{2} I$ be the Lyapunov function, where $c_{1}$ and $c_{2}$ are constants

$$
\begin{aligned}
& \frac{d L}{d t}=c_{1} \frac{d E}{d t}+c_{2} \frac{d I}{d t}(*) \\
& \Rightarrow \frac{d L}{d t}=\frac{\sigma}{(\mu+\sigma)(\mu+\xi+\gamma)} \frac{d E}{d t}+\frac{1}{\mu+\xi+\gamma} \frac{d I}{d t} \\
& =\frac{\sigma}{(\mu+\sigma)(\mu+\xi+\gamma)}[\lambda \beta S I+\lambda \beta(1-\xi) V I-(\mu+\sigma) E]+\frac{1}{(\mu+\xi+\gamma)}[\sigma E-(\mu+\xi+\gamma) I] \\
& =\frac{\sigma}{(\mu+\sigma)(\mu+\xi+\gamma)}[\lambda \beta S I+\lambda \beta(1-\xi) V I]-\frac{\sigma E}{(\mu+\xi+\gamma)}+\frac{\sigma E}{(\mu+\xi+\gamma)}-I
\end{aligned}
$$

At the D. F. E $\lambda=1$

$$
\begin{aligned}
& \Rightarrow \frac{d L}{d t}=\frac{\sigma \beta}{(\mu+\sigma)(\mu+\xi+\gamma)}\left[\frac{\wedge \omega+\wedge \mu(1-\rho)}{\mu(\mu+\omega)}+\frac{(1-\xi) \wedge \rho}{(\mu+\omega)}\right] I-I \\
& \frac{d L}{d t}=\frac{\sigma \beta \wedge[\omega+\mu(1-\rho \xi)] I}{\mu(\mu+\sigma)(\mu+\omega)(\mu+\xi+\gamma)}-I
\end{aligned}
$$

But

$$
\begin{gathered}
\frac{\sigma \beta \wedge[\omega+\mu(1-\rho \xi)]}{\mu(\mu+\sigma)(\mu+\omega)(\mu+\xi+\gamma)}=R_{0} \\
\Rightarrow \frac{d L}{d t}=R_{0} I-I \\
\frac{d L}{d t}=I\left(R_{0}-1\right) \\
\frac{d L}{d t} \leq I\left(R_{0}-1\right)
\end{gathered}
$$

Hence by Lyapunev-Lasalle asymptotic stability theorem in [24], when $R_{0} \leq 1$ it implies that the disease-free equilibrium state $\left(E^{*}\right)$ is globally asymptotically stable in $R_{+}^{5}$.

Because $\left(E^{*}\right)$ is the only point in $R^{5}$ where $\frac{d L}{d t}=\frac{d N}{d t}=0$ that is the derivatives of both $N$ and $L$ are equal to zero, then is $\left(E^{*}\right)$ unique.

This completes the proof.

Remarks: $R_{0} \leq 1$ implies that $\frac{d L}{d t}<0$ holds everywhere in
$R_{+}^{5}$ except the points where $\frac{d L}{d t}=0$

By Lyapunov-Lasalle asymptotic stability theorem, this implies that the largest invariant set of the system is globally asymptotically stable.

The global stability of the D. F. E. state means that; any initial level of S. pneumonia infection, the infection will gradually die out from the population when $R_{0} \leq 1$.

$R_{0}>1$ means that one infected individual living in an entirely Susceptible population will cause an average more than one infected individual in the next generation; in this case, S. pneumonia invades such a population and persists.

Basic reproduction rate $R_{0}$ is greater than 1 implies that the D. F. E. is unstable.

\subsection{Global Stability of Endemic Steady State}

Theorem 3.4: The unique endemic equilibrium of mode (1) given by $E^{* *}$ is globally asymptotically stable in $\Omega$ if $R_{0}>1$.

Proof:

Consider the following non-linear Lyaponuv function 


$$
L=c_{1}\left[S-S^{* *}-S^{* *} \ln \left(\frac{S}{S^{* *}}\right)+E-E^{* *}-E^{* *} \ln \left(\frac{E}{E^{* *}}\right)\right]+c_{2}\left[V-V^{* *}-V^{* *} \ln \left(\frac{V}{V^{* *}}\right)\right]+c_{3}\left[I-I^{* *}-I^{* *} \ln \left(\frac{I}{I^{* *}}\right)\right]
$$

With the Lyaponuv

$$
\begin{aligned}
& \frac{d L}{d t}=c_{1}\left[\left(1-\frac{S^{* *}}{S}\right) S^{*}+\left(1-\frac{E^{* *}}{E}\right){ }^{*}\right]+c_{2}\left[\left(1-\frac{V^{* *}}{V}\right){ }^{*}\right]+c_{3}\left[\left(1-\frac{I^{* *}}{I}\right)^{*} I\right] \\
& \frac{d L}{d t}=c_{1}\left[\left(1-\frac{S^{* *}}{S}\right)((1-\rho) \wedge+\omega V+\rho R-\lambda \beta S I-\mu S)+\left(1-\frac{E^{* *}}{E}\right)(\lambda \beta S I+\lambda \beta(1-\xi) V I-(\mu+\omega) E)\right] \\
& +c_{2}\left[\left(1-\frac{V^{* *}}{V}\right)(\rho \wedge-\lambda \beta(1-\xi) V I-(\mu+\omega) V)\right] \\
& +c_{3}\left[\left(1-\frac{I^{* *}}{I}\right)(\sigma E-(\mu+\xi+\gamma) I)\right]
\end{aligned}
$$

At the steady state;

$$
\begin{aligned}
& (1-\rho) \wedge=\lambda \beta S^{* *} I^{* *}-\omega V^{* *}-\rho R^{* *}+\mu S^{* *} \\
& \rho \wedge=\lambda \beta(1-\xi) V^{* *} I^{* *}+(\mu+\omega) V^{* *}
\end{aligned}
$$

That implies that;

$$
\begin{aligned}
& \frac{d L}{d t}=c_{1}\left[\left(1-\frac{S^{* *}}{S}\right)\left(\lambda \beta S^{* *} I^{* *}-\omega V^{* *}-\rho R^{* *}+\mu S^{* *}+\omega V+\rho R-2 \beta S I-\mu S\right)+\left(1-\frac{E^{* *}}{E}\right)(\lambda \beta S I+\lambda \beta(1-\xi) V I-(\mu+\omega) E)\right] \\
& +c_{2}\left[\left(1-\frac{V^{* *}}{V}\right)\left(\lambda \beta(1-\xi) V^{* *} I^{* *}+(\mu+\omega) V^{* *}-\lambda \beta(1-\xi) V I-(\mu+\omega) V\right)\right] \\
& +c_{3}\left[\left(1-\frac{I^{* *}}{I}\right)(\sigma E-(\mu+\xi+\gamma) I)\right]
\end{aligned}
$$

Collecting all the terms without $* *$ in the infected classes and equating them to zero $(0)$

Letting

$$
\begin{aligned}
& -c_{1} \lambda \beta S I-c_{1} \lambda \beta S^{* *} I+c_{1} \lambda \beta(1-\xi) V I-c_{2} \lambda \beta(1-\xi) V I+c_{2} \lambda \beta(1-\xi) V^{* *} I+c_{3} \sigma E=0 \\
& c_{1}\left[\left[\lambda \beta(1-\xi) V+\lambda \beta S^{* *}\right]+c_{2}\left[\lambda \beta(1-\xi) V^{* *}-\lambda \beta(1-\xi) V\right] I\right] \\
& c_{1}=\frac{\left[\lambda \beta(1-\xi) V-\lambda \beta(1-\xi) V^{* *}\right] c_{2}}{\lambda \beta\left[(1-\xi) V+S^{* *}\right]} \\
& c_{1}=\frac{(1-\xi)\left(V-V^{* *}\right) c_{2}}{(1-\xi) V+S^{* *}} \\
& c_{2}=(1-\xi) V+S^{* *} \Rightarrow c_{1}=(1-\xi)\left(V-V^{* *}\right)
\end{aligned}
$$

Also 


$$
\begin{aligned}
& c_{3} \sigma E=0 ; \sigma \neq 0 \Rightarrow c_{3}=0 \\
& L=(1-\xi)\left(V-V^{* *}\right)\left[S-S^{* *}-S^{* *} \ln \left(\frac{S}{S^{* *}}\right)+E-E^{* *}-E^{* *} \ln \left(\frac{E}{E^{* *}}\right)\right]+\left[(1-\xi) V+S^{* *}\right]\left[V-V^{* *}-V^{* *} \ln \left(\frac{V}{V^{* *}}\right)\right] \\
& \frac{d L}{d t}=(1-\xi)\left(V-V^{* *}\right)\left[S-\frac{S^{* *} *}{S} S+E-\frac{E^{* *} *}{E} E\right]+(1-\xi) V\left[*-V^{* *} V^{*}\right]+S^{* *}\left[\begin{array}{l}
* \\
V-\frac{V^{* *}}{V} V
\end{array}\right] \\
& =(1-\xi) V\left[\stackrel{*}{S}-\frac{S^{* *}}{S} S+{ }^{*} E-\frac{E^{* *}}{E} E+\stackrel{*}{V}^{*}-\frac{V^{* *}}{V} V\right]-(1-\xi) V^{* *}\left[\stackrel{*}{S}-\frac{S^{* *}}{S} \stackrel{*}{*}^{*}+E^{*} \frac{E^{* *}}{E} E\right]+S^{* *}\left[\stackrel{*}{V}-\frac{V^{* *}}{V} V\right] \\
& =(1-\xi) V\left[\begin{array}{l}
(1-\rho) \wedge+\omega V+\rho R-\lambda \beta S I-\mu S-\frac{S^{* *}}{S}((1-\rho) \wedge+\omega V+\rho R-\lambda \beta S I-\mu S) \\
+\lambda \beta S I+\lambda \beta(1-\xi) V I-(\mu+\sigma) E-\frac{E^{* *}}{E}(\lambda \beta S I-\lambda \beta(1-\xi) V I-(\mu+\sigma) E) \\
+\rho \wedge-\lambda \beta(1-\xi) V I-(\mu+\omega) V-\frac{V^{* *}}{V}(\rho \wedge-\lambda \beta(1-\xi) V I(\mu+\omega) V)
\end{array}\right] \\
& -(1-\xi) V^{* *}\left[\begin{array}{c}
(1-\rho) \wedge+\omega V+\rho R-\lambda \beta S I-\mu S-\frac{S^{* *}}{S}((1-\rho) \wedge+\omega V+\rho R-\lambda \beta S I-\mu S) \\
+\lambda \beta S I+\lambda \beta(1-\xi) V I-(\mu+\sigma) E-\frac{E^{* *}}{E}(\lambda \beta S I+\lambda \beta(1-\xi) V I-(\mu+\sigma) E)
\end{array}\right] \\
& +S^{* *}\left[\rho \wedge-\lambda \beta(1-\xi) V I-(\mu+\omega) V-\frac{V^{* *}}{V}(\rho \wedge-\lambda \beta(1-\xi) V I-(\mu+\omega) V)\right] \\
& =(1-\xi)\left[\begin{array}{l}
\lambda \beta S^{* *} I^{* *}-\omega V^{* *}-\rho R^{* *}+\mu S^{* *}+\omega V+\rho R-\lambda \beta S I-\mu S \\
-\frac{S^{* *}}{S}\left(\lambda \beta S^{* *} I^{* *}-\omega V^{* *}-\rho R^{* *}+\mu S^{* *}+\omega V+\rho R-\lambda \beta S I-\mu S\right)+\lambda \beta S I+\lambda \beta(1-\xi) V I-(\mu+\sigma) E \\
-\frac{E^{* *}}{E}(\lambda \beta S I+\lambda \beta(1-\xi) V I-(\mu+\sigma) E)+\lambda \beta(1-\xi) V^{* *} I^{* *}+(\mu+\omega) V^{* *}-\lambda \beta(1-\xi) V I-(\mu+\omega) V \\
-\frac{V^{* *}}{V}\left(\lambda \beta(1-\xi) V^{* *} I^{* *}+(\mu+\omega) V^{* *}-\lambda \beta(1-\xi) V I-(\mu+\omega) V\right)
\end{array}\right] \\
& -(1-\xi) V^{* *}\left[\begin{array}{l}
\lambda \beta S^{* *} I^{* *}-\omega V^{* *}-\rho R^{* *}+\mu S^{* *}+\omega V+\rho R-\lambda \beta S I-\mu S \\
-\frac{S^{* *}}{S}\left(\lambda \beta S^{* *} I^{* *}-\omega V^{* *}-\rho R^{* *}+\mu S^{* *}+\omega V+\rho R-\lambda \beta S I-\mu S\right) \\
+\lambda \beta S I+\lambda \beta(1-\xi) V I-(\mu+\sigma) E-\frac{E^{* *}}{E}(\lambda \beta S I+\lambda \beta(1-\xi) V I-(\mu+\sigma) E)
\end{array}\right] \\
& +S^{* *}\left[\begin{array}{l}
\lambda \beta(1-\xi) V^{* *} I^{* *}+(\mu+\omega) V^{* *}-\lambda \beta(1-\xi) V I-(\mu+\omega) V \\
-\frac{V^{* *}}{V}\left(\lambda \beta(1-\xi) V^{* *} I^{* *}+(\mu+\omega) V^{* *}-\lambda \beta(1-\xi) V I-(\mu+\omega) V\right)
\end{array}\right]
\end{aligned}
$$

Collecting all the terms with $* *$ in the infected classes including all the $\mu S^{* *},(\mu+\sigma) E,(\mu+\sigma) E^{* *},(\mu+\omega) V$ and $(\mu+\omega) V^{* *}$ 


$$
\begin{aligned}
&=(1-\xi)\left[\begin{array}{l}
\left.\lambda \beta V S^{* *} I^{* *}-\lambda \beta \frac{V S^{* 2^{2}}}{S} I^{* *}+\lambda \beta \frac{V^{* *} S^{* * 2}}{S} I^{* *}-\lambda \beta \frac{V^{* * 2} S^{* *}}{V} I^{* *}\right] \\
+\lambda \beta(1-\xi) V V^{* *} I^{* *}-\lambda \beta(1-\xi) V^{* * 2} I^{* *}
\end{array}\right] \\
&+(1-\xi)\left[\lambda \beta S V^{* *} I \frac{E^{* *}}{E}-\lambda \beta S I V \frac{E^{* *}}{E}+\lambda \beta(1-\xi) V V^{* *} I \frac{E^{* *}}{E}-\lambda \beta(1-\xi) V^{2} I \frac{E^{* *}}{E}\right] \\
&+(1-\xi)\left[\left(2 V \mu S^{* *}-V \mu S-\frac{V \mu S^{* * 2}}{S}\right)-\left(2 \mu V^{* *} S^{* *}-\mu V^{* *} S-\frac{\mu V^{* *} S^{* * 2}}{S}\right)\right] \\
&+(1-\xi)\left[V(\mu+\sigma)\left(E^{* *}-E\right)+V^{* *}(\mu+\sigma)\left(E-E^{* *}\right)\right] \\
&+(1-\xi)\left[(\mu+\omega)\left(2 V V^{* *}-V^{2}-V^{* * 2}\right)\right]+(\mu+\omega)\left[2 S^{* *} V^{* *}-V S^{* *}-\frac{V^{* * 2} S^{* *}}{V}\right] \\
&=\lambda \beta(1-\xi) S^{* *} V^{* *} I^{* *}\left[\frac{V}{V^{* *}}-\frac{V}{V^{* *}} \frac{S^{* *}}{S}+\frac{S^{* *}}{S}-\frac{V^{* *}}{V}+(1-\xi) \frac{V}{S^{* *}}-(1-\xi) \frac{V^{* *}}{S^{* *}}\right] \\
&+\lambda \beta(1-\xi) S V I\left[\frac{V^{* *}}{V}-1+(1-\xi) \frac{V^{* *}}{S}-(1-\xi) \frac{V}{S}\right] \frac{E^{* *}}{E} \\
&+\mu(1-\xi) V S^{* *}\left[2-\frac{S}{S^{* *}}\right]-\mu(1-\xi) V^{* * *} S^{* *}\left[2-\frac{S}{S^{* *}}-\frac{S^{* *}}{S}\right]+(1-\xi)(\mu+\sigma) V E^{* *}\left[1-\frac{E}{E^{* *}}\right] \\
&+(1-\xi)(\mu+\sigma) V^{* *} E^{* *}\left[2-\frac{V}{V^{* *}}-\frac{V^{* *}}{V}\right]+(\mu+\omega) V^{* *} S^{* *}\left[2-\frac{V}{V^{* *}}-\frac{V^{* *}}{V}\right]
\end{aligned}
$$

It can be shown that

$$
(1-\xi) \frac{V}{S^{* *}}-\frac{(1-\xi) V^{* *}}{S^{* *}}=0
$$

And

$$
(1-\xi) \frac{V^{* *}}{S}-(1-\xi) \frac{V}{S}=0
$$

Hence we have

$$
\begin{aligned}
& \frac{d L}{d t} \leq \mu(1-\xi) V S^{* *}\left[2-\frac{S}{S^{* *}}-\frac{S^{* *}}{S}\right]-\mu(1-\xi) V^{* *} S^{* *}\left[2-\frac{S^{* *}}{S}-\frac{S}{S^{* *}}\right] \\
& +(1-\xi)(\mu+\sigma) V E^{* *}\left[1-\frac{E}{E^{* *}}\right]+(1-\xi)(\mu+\sigma) V^{* *} E^{* *}\left[\frac{E}{E^{* *}}-1\right] \\
& +(1-\xi)(\mu+\omega) V V^{* *}\left[2-\frac{V}{V^{* *}}-\frac{V^{* *}}{V}\right]+(\mu+\omega) V^{* *} S^{* *}\left(2-\frac{V}{V^{* *}} \frac{V^{* *}}{V}\right)
\end{aligned}
$$

Finally, since the arithmetic mean exceeds the geometric mean, then

$$
\begin{aligned}
& \left(2-\frac{S^{* *}}{S}-\frac{S}{S^{* *}}\right) \leq 0 \\
& \left(2-V-\frac{V}{V^{* *}}\right) \leq 0
\end{aligned}
$$

Furthermore, since all the model parameters are non-negative, it follows that 
$\frac{d L}{d t} \leq 0$ for $R_{0}>1$. Thus $L$ is aLyapunov function of system (4) on $\Omega$.

Therefore, it follows from Lasalle's Invariance Principle that

$$
\begin{aligned}
& \lim _{t \rightarrow \infty} S(t)=S^{* *} \\
& \lim _{t \rightarrow \infty} V(t)=V^{* *} \\
& \lim _{t \rightarrow \infty} E(t)=E^{* *} \\
& \lim _{t \rightarrow \infty} I(t)=I^{* *} \\
& \lim _{t \rightarrow \infty} R(t)=R^{* *}
\end{aligned}
$$

\subsection{Basic Concept of Variational Iteration Method}

We consider the general nonlinear system

$$
L[u(x)]+N[u(x)]=\psi(x)
$$

Where $\mathrm{L}$ is linear operator, $\mathrm{N}$ is nonlinear operator and $\psi(x)$ is a given continuous function.

According to the variational iteration method He (1999) and He et al. (2006), we can construct a correction functional in the form;

$$
u_{k+1}(x)=u_{k}(x)+\int_{0}^{t} \lambda(s)\left[L\left[u_{k}(s)\right]+N\left[\tilde{u}_{k}(s)\right]-\psi(x)\right] d s
$$

Where $u_{0}(x)$ is an initial approximation with possible unknowns, $\lambda$ is the Lagrange multiplier which can be determined optimally via variational theory, the subscript $k$ denotes the $k^{\text {th }}$ approximation and $\tilde{u}_{k}$ is considered as a restricted variation such that $\delta \tilde{u}_{k}=0$. It is shown that this method is very effective and easy for a linear problem, its exact solution can be obtained by only the first iteration because $\lambda$ can be exactly identified.

Hence to solve the above equation, we proceed by considering the stationary condition of the correction functional, then the Lagrange multiplier $\lambda$ becomes,

$$
\lambda(s)=\frac{(-1)^{m}}{(m-1) !}(s-x)^{m-1}
$$

where $\mathrm{m}$ is the highest order of the differential equation.

As a result, we have the iteration formula as;

$$
u_{k+1}(x)=u_{k}(x)+\int_{0}^{t} \frac{(-1)^{m}}{(m-1) !}(s-x)^{m-1}\left[L\left[u_{k}(s)\right]+N\left[\tilde{u}_{k}(s)\right]-\psi(x)\right] d s
$$

\subsection{Implementation of Variational Iteration Method on SVEIR Model}

First we consider the SVEIR model (4), we then apply Variational Iteration Method (VIM) by constructing the correction functional of each of the equations of the system, and we obtained the following;

$$
\begin{gathered}
S_{n+1}=S_{n}+\int_{0}^{t} \lambda_{1}(t)\left[\frac{d S_{n}}{d t}-(1-p) \Lambda-\omega V_{n}-\rho R_{n}+\frac{\beta \tilde{S}_{n} \tilde{I}_{n}}{1+\alpha I}+\mu S_{n}\right] d t \\
V_{n+1}=V_{n}+\int_{0}^{t} \lambda_{2}(t)\left[\frac{d V_{n}}{d t}-p \Lambda+\frac{\beta(1-\xi) I_{n} V_{n}}{1+\alpha I}+(\omega+\mu) V_{n}\right] d t \\
E_{n+1}=E_{n}+\int_{0}^{t} \lambda_{3}(t)\left[\frac{d E_{n}}{d t}-\frac{\beta I_{n} S_{n}}{1+\alpha I}-\frac{\beta(1-\xi) I_{n} V_{n}}{1+\alpha I}+(\sigma+\mu) E_{n}\right] d t
\end{gathered}
$$




$$
\begin{gathered}
I_{n+1}=I_{n}+\int_{0}^{t} \lambda_{4}(t)\left[\frac{d I_{n}}{d t}-\sigma E_{n}+(\mu+\varepsilon+\gamma) I_{n}\right] d t \\
R_{n+1}=R_{n}+\int_{0}^{t} \lambda_{5}(t)\left[\frac{d R_{n}}{d t}-\gamma I_{n}+(\rho+\mu) R_{n}\right] d t
\end{gathered}
$$

Where $\lambda_{i}, i=1,2, \ldots 5$ are the Lagrangemultiplier which can be identified optimally via variational Theory.

we now obtain the optimal values of $\lambda_{i}$

$$
\lambda_{i}(t)=\frac{(-1)^{m}}{(m-1) !}(t-x)^{m-1}, \quad i=1,2,3,4,5
$$

$m$ is the highest order of the differential equations.

$x$ is the compartments

Hence we have

$$
\begin{aligned}
& \lambda_{1}(t)=\frac{(-1)^{1}}{(1-1) !}(t-S)^{1-1}=\frac{-1}{0 !}(t-S)^{0}=-1 \\
& \lambda_{2}(t)=\frac{(-1)^{1}}{(1-1) !}(t-V)^{1-1}=\frac{-1}{0 !}(t-V)^{0}=-1 \\
& \lambda_{3}(t)=\frac{(-1)^{1}}{(1-1) !}(t-E)^{1-1}=\frac{-1}{0 !}(t-E)^{0}=-1 \\
& \lambda_{4}(t)=\frac{(-1)^{1}}{(1-1) !}(t-I)^{1-1}=\frac{-1}{0 !}(t-I)^{0}=-1 \\
& \lambda_{5}(t)=\frac{(-1)^{1}}{(1-1) !}(t-R)^{1-1}=\frac{-1}{0 !}(t-R)^{0}=-1
\end{aligned}
$$

Therefore, our iteration formulae become;

$$
\begin{gathered}
S_{n+1}=S_{n}-\int_{0}^{t}\left[\frac{d S_{n}}{d t}-(1-p) \Lambda-\omega V_{n}-\rho R_{n}+\frac{\beta \tilde{S}_{n} \tilde{I}_{n}}{1+\alpha I}+\mu S_{n}\right] d t \\
V_{n+1}=V_{n}-\int_{0}^{t}\left[\frac{d V_{n}}{d t}-p \Lambda+\frac{\beta(1-\xi) I_{n} V_{n}}{1+\alpha I}+(\omega+\mu) V_{n}\right] d t \\
E_{n+1}=E_{n}-\int_{0}^{t}\left[\frac{d E_{n}}{d t}-\frac{\beta I_{n} S_{n}}{1+\alpha I}-\frac{\beta(1-\xi) I_{n} V_{n}}{1+\alpha I}+(\sigma+\mu) E_{n}\right] d t \\
I_{n+1}=I_{n}-\int_{0}^{t} \lambda_{4}(t)\left[\frac{d I_{n}}{d t}-\sigma E_{n}+(\mu+\varepsilon+\gamma) I_{n}\right] d t \\
R_{n+1}=R_{n}-\int_{0}^{t} \lambda_{5}(t)\left[\frac{d R_{n}}{d t}-\gamma I_{n}+(\rho+\mu) R_{n}\right] d t
\end{gathered}
$$

With the initial approximation $S_{0}(t)=25, V_{0}(t)=20, E_{0}(t)=15, I_{0}(t)=10, R_{0}(t)=0$ which satisfy the initial conditions, to give; 


$$
\begin{gathered}
S_{1}(t)=25+7.57218 t \\
V_{1}(t)=20+1.8944104 t \\
E_{1}(t)=15+0.4470096 t \\
I_{1}(t)=10-3.8516 t \\
R_{1}(t)=0.714 t \\
S_{2}(t)=25+7.57218 t+3.428031601 t^{2}+0.0133925719 t^{3} \\
V_{2}(t)=20+1.8944104 t-0.01980016004 t^{2}+0.003283546738 t^{3} \\
E_{2}(t)=15+0.4470096 t-0.05587241364 t^{2}-0.01667611864 t^{3} \\
I_{2}(t)=10-3.8516 t+0.7758508925 t^{2} \\
R_{2}(t)=0.714 t-3.89337352 t^{2} \\
S_{3}(t)=25+7.57218 t+3.428031601 t^{2}-10.95779782 t^{3}+0.02528127049 t^{4} \\
-0.0007185722909 t^{5}-0.000002385690683 t^{6} \\
V_{3}(t)=20+1.8944104 t-0.01980016004 t^{2}-0.003199072356 t^{3}-0.0005840314321 t^{4} \\
0.0000075626666608 t^{5}-5.73214805 \times 10^{-7} t^{6} \\
E_{3}(t)=15+0.4470096 t-0.05587241364 t^{2}+0.01507381852 t^{3}-0.001898403052 t^{4} \\
+0.0007110096243 t^{5}+0.000002958908163 t^{6}
\end{gathered}
$$

\subsection{Basic Concept and Implementation of ADM on SVEIR Model}

In this section, we determine the solution of model (4) using Adomain Decomposition Method (ADM). Let us consider system (4) in an operator form;

$$
\left.\begin{array}{l}
L S=(1-\rho) \Lambda+\omega \Lambda+\rho \Lambda-\lambda \beta S I-\mu S \\
L V=\rho \Lambda-\lambda \beta(1-\xi) V I-(\mu+\omega) V \\
L E=\lambda \beta S I+\lambda \beta(1-\xi) V I-(\mu+\sigma) E \\
L I=\sigma E-(\mu+\varepsilon+\gamma) I \\
L R=\gamma I-(\mu+\rho) R \\
\text { where } L=\frac{d}{d t}
\end{array}\right\}
$$

By applying the inverse operator $L^{-1}=\int_{0}^{t}() d$.$t on both sides of (* *)$, we have 


$$
\left\{\begin{array}{l}
S(t)=S(0)+(1-p) \Lambda t+\omega \int_{0}^{t} V d t+\rho \int_{0}^{t} R d t-\lambda \beta \int_{0}^{t} S I d t-\mu \int_{0}^{t} S d t \\
V(t)=V(0)+p V t-\lambda \beta(1-\xi) \int_{0}^{t} V I d t-(\mu+\omega) \int_{0}^{t} V d t \\
E(t)=E(0)+\lambda \beta \int_{0}^{t} S I d t+\lambda \beta(1-\xi) \int_{0}^{t} V I d t-(\mu+\sigma) \int_{0}^{t} E d t \\
I(t)=I(0)+\sigma \int_{0}^{t} E d t-(\mu+\varepsilon+\gamma) \int_{0}^{t} I d t \\
R(t)=R(0)+\gamma \int_{0}^{t} I d t-(\mu+\rho) \int_{0}^{t} R d t
\end{array}\right\}
$$

Applying Adomain Decomposition Method, the solution of the equation (35) above becomes sum of the following series; The linear terms

$$
S=\sum_{n=0}^{\infty} S_{n} ; V=\sum_{n=0}^{\infty} V_{n} ; E=\sum_{n=0}^{\infty} E_{n} ; I=\sum_{n=0}^{\infty} I_{n} ; R=\sum_{n=0}^{\infty} E_{n}
$$

And we approximate the non-linear terms as follows

$$
\begin{aligned}
& S I=\sum_{n=0}^{\infty} A_{n}\left(S_{0}, \ldots, S_{n ; I_{0}}, \ldots, I_{n}\right) \\
& V I=\sum_{n=0}^{\infty} B_{n}\left(V_{0}, \ldots, V_{n} ; I_{0}, \ldots, I_{n}\right)
\end{aligned}
$$

Where

$$
A_{n}=\frac{1}{n !}\left[\frac{d^{n}\left(\sum_{n=0}^{\infty} S_{k} \lambda^{k}\right)\left(\sum_{n=0}^{\infty} I_{k} \lambda^{k}\right)}{d \lambda^{n}}\right] \lambda=0
$$

and

$$
B_{n}=\frac{1}{n !}\left[\frac{d^{n}\left(\sum_{n=0}^{\infty} S_{n} \lambda^{k}\right)\left(\sum_{n=0}^{\infty} I_{n} \lambda^{k}\right)}{d \lambda^{k}}\right]_{\lambda=0}
$$

Are the Adomain polynomials

Now, substituting $A_{n}, B_{n}$ and the linear terms in (20), we have

Hence from the above equation (21) we define the following initial conditions and recursive formula 


$$
\begin{aligned}
S_{0}=S(0)+(1-p) \wedge t, V_{0}=V(0)+p \wedge t, E_{0}=E(0), I_{0}=I(0), R_{0}=R(0) & \\
S_{n+1} & =\omega \int_{0}^{t} V_{n} d t+\rho \int_{0}^{t} R_{n} d t-\lambda \beta \int_{0}^{t} A_{n} d t-\mu \int_{0}^{t} S_{n} d t \\
S_{n+1} & =\lambda \beta(1-\xi) \int_{0}^{t} B_{n} d t-(\mu+\omega) \int_{0}^{t} V_{n} d t \\
E_{n+1} & =\lambda \beta \int_{0}^{t} A_{n} d t+\lambda \beta(1-\xi) \int_{0}^{t} B_{n} d t-(\mu+\sigma) \int_{0}^{t} E_{n} d t \\
I_{n+1} & =\sigma \int_{0}^{t} E_{n} d t-(\mu+\xi+r) \int_{0}^{t} I_{n} d t \\
R_{n+1} & =r \int_{0}^{t} I_{n} d t-(\mu+\rho) \int_{0}^{t} R_{n} d t
\end{aligned}
$$

We compute the Adomain polynomial with help of Maple; and obtained

$$
\begin{aligned}
A_{0} & =S_{0} I_{0} \\
A_{1} & =S_{0} I_{1}+S_{1} I_{0} \\
A_{2} & =S_{0} I_{2}+S_{1} I_{1}+S_{2} I_{0} \\
A_{3} & =S_{0} I_{3}+S_{1} I_{2}+S_{2} I_{1}+S_{3} I_{0} \\
A_{4} & =S_{0} I_{4}+S_{1} I_{3}+S_{2} I_{2}+S_{3} I_{1}+S_{4} I_{0} \\
A_{5} & =S_{0} I_{5}+S_{1} I_{4}+S_{2} I_{3}+S_{3} I_{2}+S_{4} I_{1}+S_{5} I_{0} \\
B_{0} & =V_{0} I_{0}, \\
B_{1} & =V_{0} I_{1}+V_{1} I_{0} \\
B_{2} & =V_{0} I_{2}+V_{1} I_{1}+V_{2} I_{0} \\
B_{3} & =V_{0} I_{3}+V_{1} I_{3}+V_{2} I_{2}+V_{3} I_{1}+V_{4} I_{0} \\
B_{4} & =V_{0} I_{5}+V_{1} I_{4}+V_{2} I_{3}+V_{3} I_{2}+V_{4} I_{1}+V_{5} I_{0} \\
& S_{1}=0.8926 t+0.059216635 t^{2} \\
& V_{1}=-1.5160096 t+0.1 .1292557365 t^{2} \\
& E_{1}=0.4470098 t+0.06903010054 t^{2} \\
& I_{1}=-3.85160 t \\
R_{1} & =0.7140 t \\
S_{2} & =3.368814965 t^{2}+0.008862406378 t^{3} \\
V_{2} & =0.1094555764 t^{2}+0.009177100611 t^{3} \\
E_{2} & =-0.1249025142 t^{2}-0.01829162969 t^{3} \\
I_{2} & =0.7758508926 t^{2}+0.0002521899673 t^{3} \\
R_{2} & =-3.493373520 t^{2}
\end{aligned}
$$




$$
\begin{aligned}
& S_{3}=-10.96666022 t^{3}-0.001596915911 t^{4}-4.641197940 \times 10^{-7} t^{4} \\
& V_{3}=-0.01237617297 t^{3}-0.001236686070 t^{4}-2.322281588 \times 10^{-7} t^{5} \\
& E_{3}=0.03336544822 t^{3}+0.002883733653 t^{4}+6.963479520 \times 10^{-7} t^{5} \\
& I_{3}=-0.1043168833 t^{3}-0.00007543893886 t^{4} \\
& R_{3}=11.04999716 t^{3}+0.00002531987271 t^{4}
\end{aligned}
$$

\section{Simulation (Numerical Solution) and Results}

Here in this section, we present, the numerical simulation using some estimated parameters from the literature and others from noted secondary sources to showcase the analytical results. The simulation of the S. Pneumonia was

\begin{tabular}{|c|c|c|c|}
\hline Symbols \& Parameters & Description & Value & Source \\
\hline $\mathrm{S}$ & Susceptible Individuals & 25 & Estimated \\
\hline V & Vaccinated Individuals & 20 & Estimated \\
\hline I & Infectious individuals & 10 & Estimated \\
\hline $\mathrm{R}$ & Recovered individuals & 0 & Estimated \\
\hline$\mu$ & Per capita natural mortality rate & 0.2 & Estimated \\
\hline$\beta$ & Contact rate & 0.0287 & Mohammed and Tumwiine (2018) \\
\hline$\xi$ & Effectiveness of vaccine & 0.3 & Estimated \\
\hline $\mathrm{p}$ & Fraction of recruited individuals & 0.32 & Mohammed and Tumwiine (2018) \\
\hline$\sigma$ & Infectious rate of exposed individuals & 0.01096 & Mohammed and Tumwiine (2018) \\
\hline$\gamma$ & recovery rate of infected individuals & 0.0714 & Mohammed and Tumwiine (2018) \\
\hline$\omega$ & The rate at which vaccines wane & 0.0621 & Mohammed and Tumwiine (2018) \\
\hline$\Lambda$ & Recruitment rate into the susceptible compartment & 10.09 & Mohammed and Tumwiine (2018) \\
\hline$\alpha$ & $\begin{array}{l}\text { Proportion of antibody produced by individual in response to the incidence } \\
\text { of infection }\end{array}$ & 2 & Liu and Yang (2012) \\
\hline$\rho$ & Per capita rate of recovered individuals going back to the Susceptible class & 9.4 & Mohammed and Tumwiine (2018) \\
\hline
\end{tabular}
done using MATLAB with the sole aim of investigating the effect or the contribution of the different parameters to the spread of the S. Pneumonia infection and their mitigation.

Table 2. Parameter estimates for S. pneumonia model under interventions.

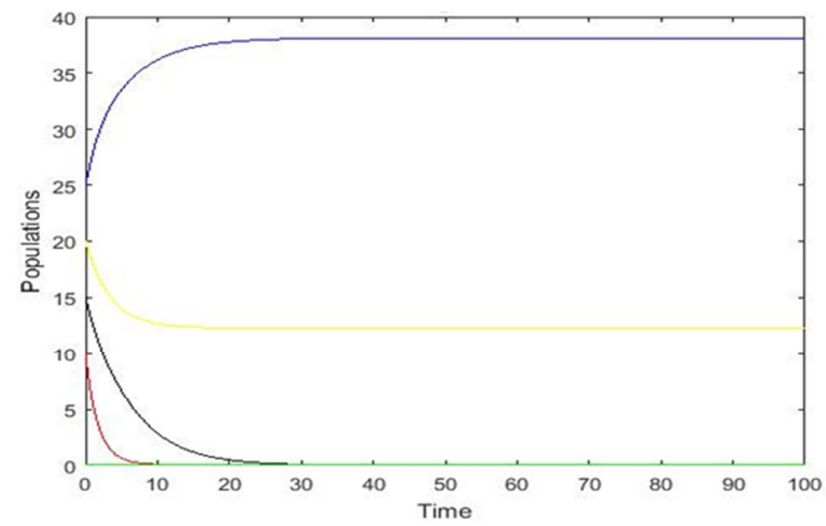

Figure 2. Plot of Population against time.

The graph below shows the trend of all the population against time when the basic reproduction number is less than one. The result show that; as can be seen from that the infected class I (t) is drastically reduces to zero (0) in the long run when the basic reproduction number is less than unity (that is $\Re_{0}=0.1154<1$ ). While the vaccinated $\mathrm{V}(\mathrm{t})$ and susceptible $S(t)$ remains in the population.

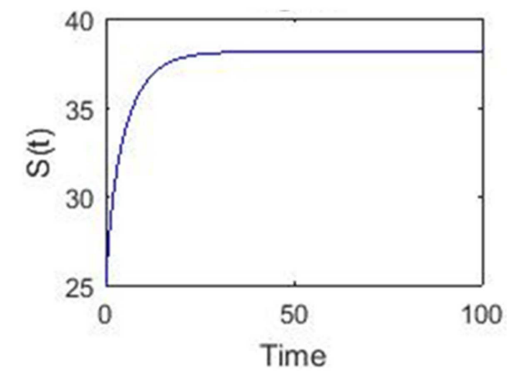

Figure 3. Susceptible Population againsttme.

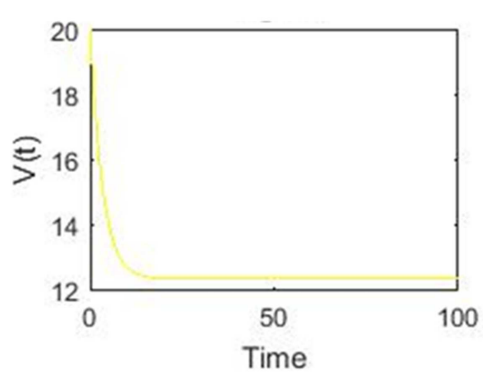

Figure 4. Vaccinated Pop. against time. 


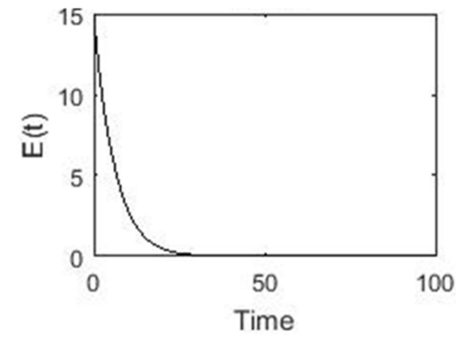

Figure 5. Plot of Exposed Pop against time.

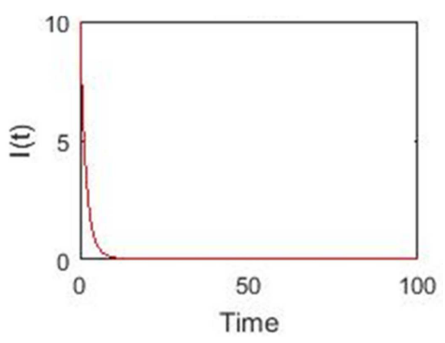

Figure 6. Plot of InfectedPop. against time.

The graphs above explicitly describe the trend of each of the classes against time when the basic reproduction number is less than unity. It is clear from the above trend that both the exposed population and the infected population are reduced to zero. This shows that our system is stable when the basic reproduction number is less than one (1).

While the vaccinated population decrease but remain constant at a particular time. The explanation to this is as a result of fear and lack of awareness of the vaccination campaign against $\mathrm{S}$. pneumonia. But after sometimes people are aware of the vaccination which maintains the population at that static level.

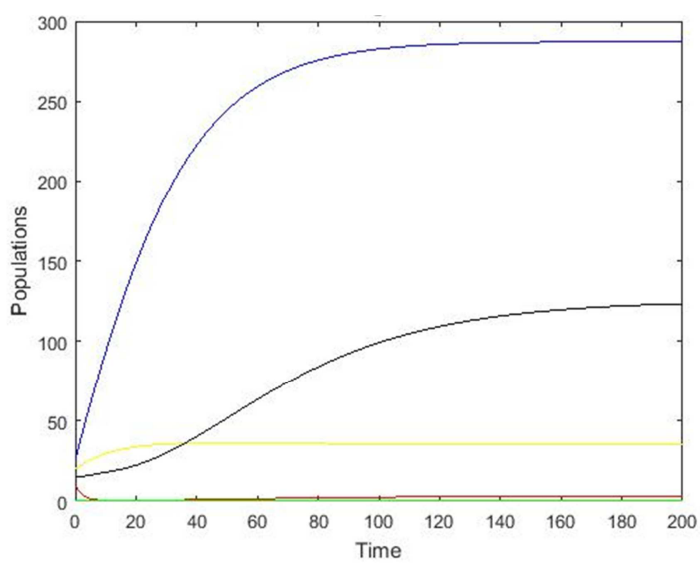

Figure 7. Plot of Population against time.

Here in this graph we have the five classes and their trend over time when $\mathfrak{R}_{0}=1.1864>1$, it was revealed from the simulation that the infected population decreases but did not get to zero, which means that there are individuals who are still living with S. Pneumonia in the population. Our simulation further shows that within the period of 10days, the number of infected individual reduces drastically and then increases within 45 to 50 day slowly.

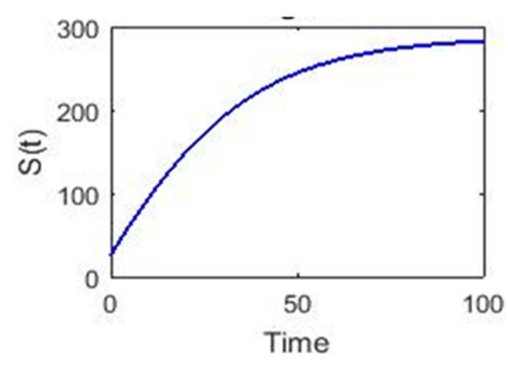

Figure 8. Susceptible Popu. Againsttime.

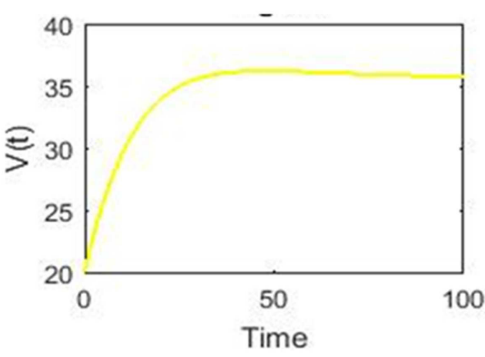

Figure 9. Vaccinated Pop. Againsttime.

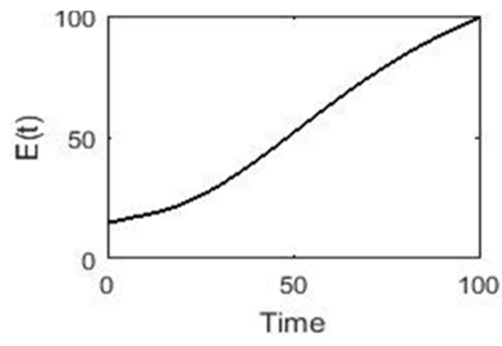

Figure 10. Exposed Pop. Against time.

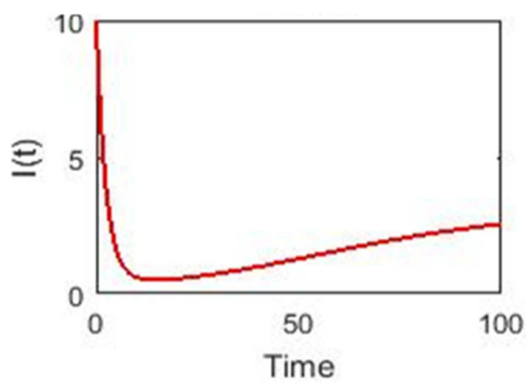

Figure 11. Infected Popu. Against time.

In the above simulation, we describe the trend of each of the classes to understand exactly what happened in Figure 9 and it reveals that, if the basic reproduction is greater than one, S. pneumonia becomes endemic in the population and persist since the exposed and infected classes were not reduced to zero, see Figure 10 and Figure 9.

Here we simulate the effect of proportion of antibody induced by the vaccine. Our simulation reveals that increase in $\alpha$ which is proportion of antibody produced by individual in response to the incidence of Infection, reduces the infected population. 

Pneumonia with Saturated Incidence Force of Infection

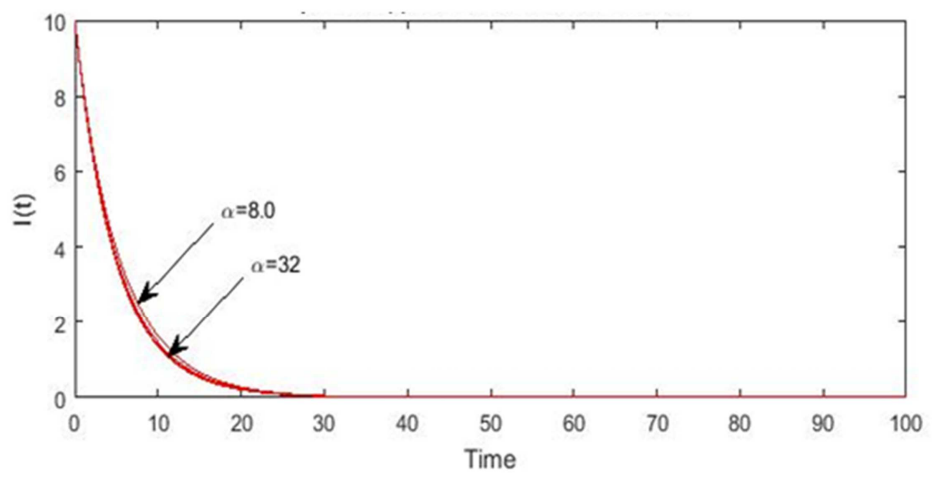

Figure 12. Plot of Infected population against time for different values $\alpha$.

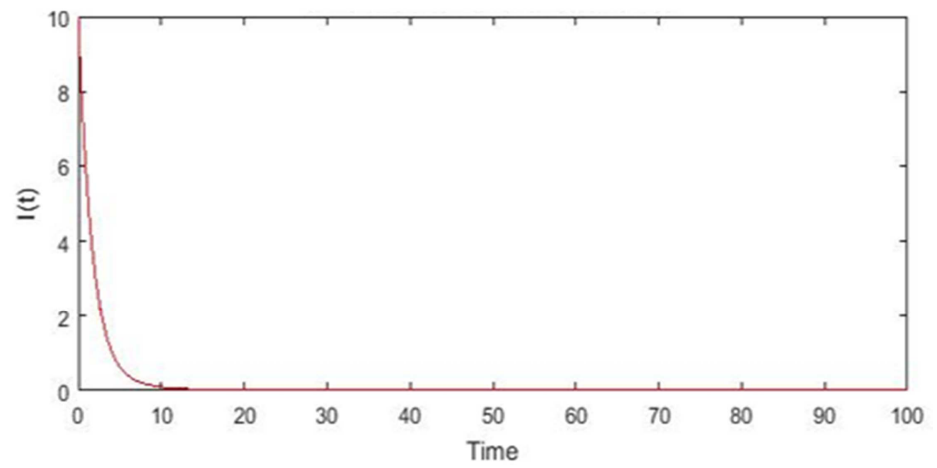

Figure 13. Plot of Infected population aginst time for different values of $\omega$.

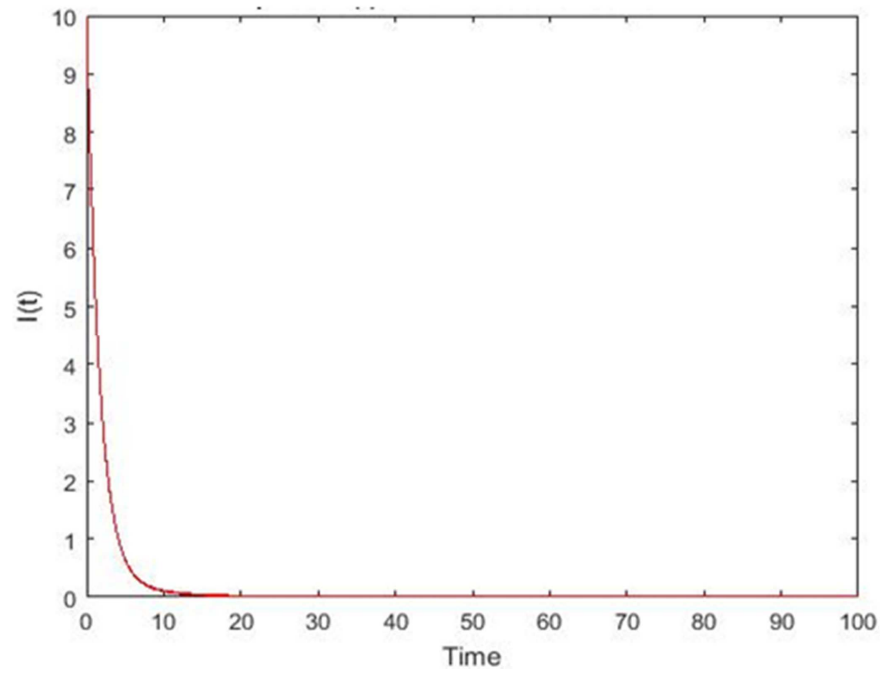

Figure 14. Plot of Infected population against time for different values of $\Lambda$.

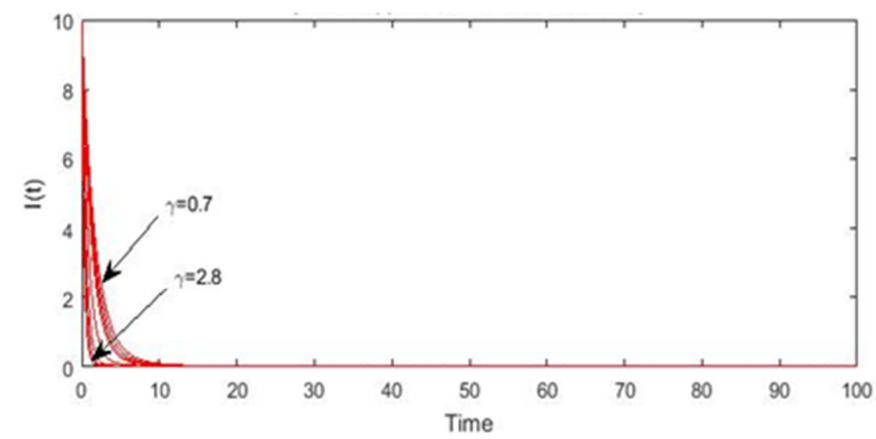

Figure 15. Plot of Infected pop againsttime for different values of $\gamma$. 
Our simulation in the two graphs above, we observed that $\omega$ and $\Lambda$ little or no variation in the infected population. The implication of this is that as more individuals with high proportion of antibody against S. pneumonia is recruited into the population, there is no need vaccinating them again, since their immune system is already in fighting against $\mathrm{S}$. pneumonia. That is to say that an individual needs vaccination before he/she will be introduced into the population. Also, we see from the simulation above that increase in recovery rate $\gamma$ reduces the number of infected individual over time.

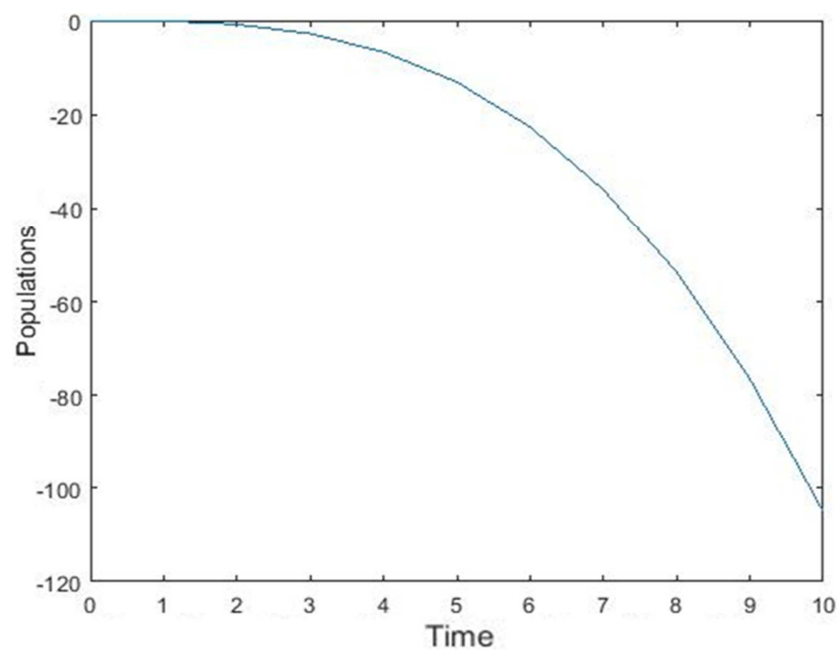

Figure 16. Plot of Population against time for VIM.

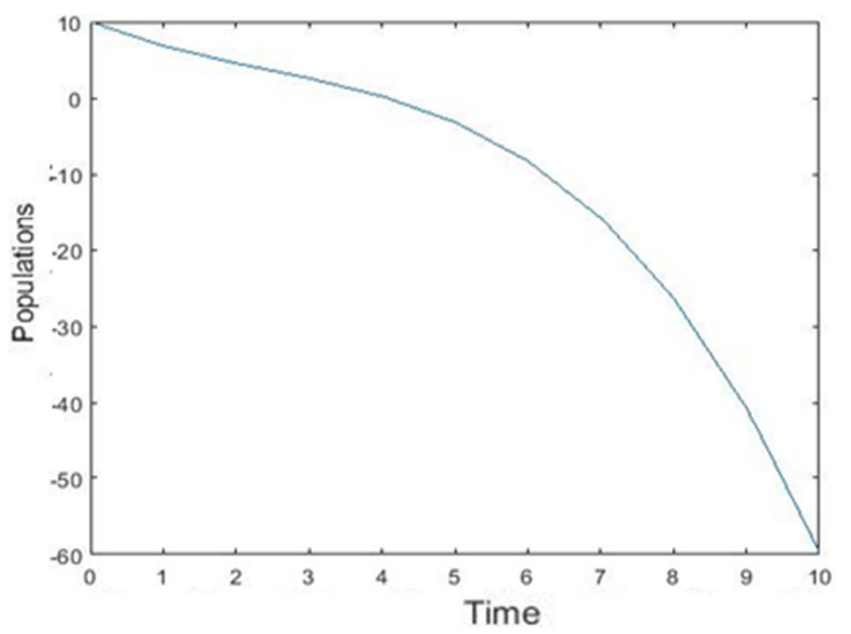

Figure 17. Plot of Population against time for ADM.

Figure 16 and Figure 17 shows the simulation for the Variational iteration Method (VIM) and Adomian Decomposition method (ADM) respectively. It was seen that in Figure 15, within the first 3 days the infection is still in the population until, the $4^{\text {th }}$ day when the number of infected individual is reduced to zero. While Figure 17 shows that from the $1^{\text {st }}$ day the infection is completely out of the population. Hence, it shows that the ADM converges faster than the VIM.

\section{Summaries, Conclusion and Recommendation}

\subsection{Summary}

In this paper, the dynamics of SVEIR model with saturated incidence force of infection and saturated vaccination function for Streptococcus pneumonia (that is, model that monitors the temporal transmission dynamics of the disease in the presence of preventive vaccine) was formulated and analyzed. The basic reproduction number that determines disease extinction and disease survival was revealed. The existing threshold conditions of all kinds of the equilibrium points are obtained and proved to be locally asymptotic stable for disease-free equilibrium using linearization method and Lypanov functional method for Endemic equilibrium. Qualitative Analysis of the model was obtained and the positive of solution obtained. It was revealed that the model is positively -invariant and attracting. Thus $\Omega$ is positively invariant. Hence, it is sufficient to consider the dynamics of the model (1) in $\Omega$. In this region, the model can be considered as been epidemiologically and mathematically well-posed, Hethcote (2000). The governing model was normalized and also Adomain Decomposition method was used to compute an approximate solution of the non-linear system of differential equations governing the model. Maple was used in carrying out the simulations (numerical solutions) of the model. Graphical results were presented and discussed to illustrate the solution of the problem. The achieved results reveal that the disease will die out within the community if the vaccination coverage is above the critical vaccination proportion. The study indicates that we should improve the efficiency and enlarge the capacity of the treatment to control the spread of disease.

\subsection{Conclusion}

It is revealed that the DFE is globally asymptotically stable while the endemic equilibrium is not feasible which implies that the disease will be eradicated out of the population If $\mathfrak{R}_{0}<1$. Furthermore, we can also see that if $\mathfrak{R}_{0}>1$ and $H>0$, then endemic equilibrium is globally asymptotically stable. In order to regulator the disease, it will be strategical to decrease the $\mathrm{BRN}$ to barest minimum. From the manifestation of $\mathrm{BRN} \mathfrak{R}_{0}$, it is obvious that the rate $\xi$ representing vaccine efficiency, the rate/transfer of individual out of the infected compartment $v_{i}^{-}$and $(1-\xi)$ revealing the rate at which the vaccine get waned directly or indirectly impact the value of $\Re_{0}$. Obviously, if $\xi, v_{i}^{-}$or $(1-\xi)$ increase, implies $\mathfrak{R}_{0}$ decreases. Therefore, the need for public health interventions to control the epidemic by ensuring these parameters $\xi, v_{i}^{-}$or $(1-\xi)$ are increased to reduce $\Re_{0}$ can never be over emphasis.

\subsection{Recommendation}

Research has shown that Invasive disease attributable to $S p$ is a major public health problem for under aged children 
irrespective of high use of the 7-valent pneumococcal conjugate vaccine (PCV7) in Nigeria and other African countries.

The epidemiology of human population especially those concerning under age children requires urgent and serious investigation so as to understand the diseases and proffer solutions that will completely eradicate it from our population. This could be achieved through

1. International partnership and research collaboration.

2. Improve funding of epidemiological research programs.

3. Advocacy and awareness creation among rural and urban communities.

4. Efficient and effective specialist vaccination centers in rural and urban communities.

5. Free and effective vaccination of under age children.

Thus the need for a greater understanding of Streptococcus Pneumonia and for more effective vaccination, treatment and control program is paramount to eradication of the infection. Therefore, we consent the effort of the US Food and Drug Administration (February 24, 2010) that licensed a new 13valent pneumococcal polysaccharide-protein conjugate vaccine (PCV13) for under age children.

Hence we would also recommend from the above knowledge:

1. Healthy children and their counterparts (both those who have completed the previous vaccine PCV7) with other health challenging issues exposing them to high risk of IPD should be routinely immunize with PCV13.

2. "Catch-up" immunization should be conducted for children behind schedule; and,

3. There should be timely treatment and vaccination for infected individuals and those with compromised immunity including newborn babies respectively.

\section{References}

[1] Acedo L, Gonzalez-Parra G, \&Arenas A. (2010) An exact global solution for the classical Epidemic model; Nonlinear Analysis: Real World Applications; 11 (3): 1819-1825.

[2] Alexander M. E, \& Moghadas S. M. (2005) Bifurcation analysis of SIRS epidemic model with generalizedIncidence, SIAM Journal on Applied Mathematics, 65 (5): 1794-181.

[3] Batt, S. L., Charalambous, B. M., Solomon, A. W., Knirsch, C., Massae, P. A., Safari, S., Sam, N. E., Everett, D., Mabey, D. C. \& Gillespie, S. H. (2003) Impact of azithromycin administration for trachoma control on the carriage of antibiotic-resistant Streptococcus pneumoniae. Antimicrobial Agents and Chemotherapy 47: 2765-2769.

[4] Blossom, D. B., Namayanja-Kaye, G., Nankya-Mutayoba, J., Mukasa, J. B., Bakka, H., Rwambuya, S., Windau, A., Bajaksouzian, S., Walker, C. J. \& Joloba, M. L. (2006) Oropharyngeal colonization by Streptococcus pneumonia among HIV-infected adults in Uganda: assessing prevalence and antimicrobial susceptibility. International Journal of Infectious Diseases 10: 458-464.

[5] Bogaert, D., De Groot, R. \& Hermans, P. (2004) Streptococcus pneumonia colonization: the key to pneumococcal disease. Lancet Infectious 4: 144-154.
[6] Centers for Disease Control and Prevention (2017) "Streptococcus pneumonia" Published on official website https://www.cdc.gov/pneumococcal/clinicians/streptococcuspneumoniae.html.

[7] Diftz K., Schenzle D. Proportionate mixing models for agedependent infection transmission J. Math. Biol., 22 (1985): 117-120.

[8] Donnelly C. A. (2003) Epidemiological determinants of spread of causal agent of severe acute respiratory syndrome in Hong Kong, The Lancet Published online May 7, 2003.

[9] Dulpl, E. (2012) Nasopharyngeal carrier rate of Streptococcus pneumonia in children: serotype distribution and antimicrobial resistance. Archieves of Iranian Medicine 15: 500.

[10] Eckalbar J. C \&Eckalbar W. L. (2011) Dynamics of an epidemic model with quadratic treatment. Nonlinear Analysis: Real World Applications. 2011; 12 (1): 320-332.

[11] Esteva L \& Matias M. (2001) A model for vector transmitted diseases with saturation incidence. Journal of Biological Systems. 2001; 9 (4): 235-245.

[12] Faden, H., Duffy, L., Wasielewski, R., Wolf, J., Krystofik, D. \& Tung, Y. (1997) Relationship between nasopharyngeal colonization and the development of otitis media in children. Journal of Infectious Diseases 175: 1440-1445.

[13] Falade, A. G. \& Ayede, A. I. (2011) Epidemiology, aetiology and management of childhood acute community-acquired pneumonia in developing counties - a review. African Journal of Medicine and Medical Sciences 40: 293-308.

[14] Hethcote H. (2000) The Mathematics of Infectious Diseases; SIAM Review; 42 (4): 599-653.

[15] Hethcote H., Zhien M, Shengbing L., (2002) "Effects of quarantine in six endemic models for infectious diseases Mathematical Biosciences, 180 (2002): 141-160, 10.1016/S0025-5564(02)00111-6.

[16] He, J. H. (1999). Variational iteration method- a kind of nonlinear analytical technique: some examples, Int. J. Nonlin. Mech., 34: 699-708.

[17] He, J. H. \& X. H. Wu (2006). Construction of solitary solutions and compacton-like solution by variational iteration method, Chos. Soltn. Frcts; 29 (1): 108-113.

[18] Hoppensteadt F. (1974) "An Age Dependent Epidemic Model" Journal of the Franklin Institute 297 (5): 325-333.

[19] Hoppensteadt F. (1974) Asymptotic stability in singular perturbation problems J. Diff. Eq, 15 (no. 3): 510-521.

[20] Jacobs, M. R. (2004) Streptococcus pneumoniae: Epidemiology and patterns of resistance. American Journal of Medicine Supplements117: 3-15.

[21] Joloba, M., Bajaksouzian, S., Plalavecino, E., Whalen, C. \& Jacobs, M. (2001a) High prevalence of carriage of antibioticresistant Streptococcus pneumoniae in children in Kampala, Uganda. International Journal of Antimicrobial Agents 17: 395-400.

[22] Kinabo G. D, Van der Ven A, Msuya, Shayo A. M, Schimana W, Ndaro A, Van Asten H, Dolmans W, Warris A, \& Hermans P (2013) Dynamics of nasopharyngeal bacterial colonization in HIV exposed young infant in Tanzania; 18 (3): 286-295. 
[23] Korobeinikov, A. (2004) Lyapunov functions and global properties for SEIR and SEIS epidemic models; Math. Med. Biol.: J. IMA., 21: 75-83.

[24] La Salle J. P. (1976) The Stability of Dynamical Systems, SIAM, No. 25.

[25] Lannelli M, Martcheva M, \& Li X.-Z., (2005) "Strain replacement in an epidemic model with super-infection and perfect vaccination," Mathematical Biosciences, 195 (1): 2346,2005 . View at Publisher View at Google Scholar View at MathSciNet · View at Scopus.

[26] Li M. Y, \&Muldowney J. S. (1996) A geometric approach to global-stability problems. SIAM Journal onMathematical Analysis; 27 (4): 1070-1083.

[27] Li, J., and Brauer, F. (2008). "Continuous-time age-structured models in population dynamics and epidemiology," in Math Epidemiol, Lecture Notes in Mathematics, eds. F. Brauer, P. van den Driessche, and J. Wu (Berlin; Heidelberg: Springer), 1945: 205-227.

[28] Li, Y. (2004). Determination of the critical concentration of neutrophils required to block bacterial growth in tissues. J. Exp. Med. 200: 613-622. doi: 10.1084/jem.2004072.

[29] Liu, X. B, \&Yang, L. J. (2012) Stability analysis of an SEIQV epidemic model with saturated incidence rate. Nonlinear Analysis: Real World Applications; 13 (6): $2671-$ 2679 .

[30] Mochan, E., Swigon, D., Ermentrout, B., Lukens, S., \& Clermont, G. (2014). A mathematical model of intrahost pneumococcal pneumonia infection dynamics in murine strains; J. Theoritical Biol. 353: 44-54. doi: 10.1016/j.jtbi.2014.02.021.

[31] McCullers, J. A., English, B. K., \& Novak, R. (2000). Isolation and characterization of vancomycin-tolerant Streptococcus pneumoniae from the cerebrospinal fluid of a patient who developed recrudescent meningitis; J. Infect. Dis. 181: 369-373. Doi: 10.1086/315216.

[32] MurrayJ. D. (2001) Mathematical Biology (2 ${ }^{\text {nd }}$, corrected Edition), Springer-Verlag, pp. 385-396.

[33] Nantanda, D. M., Hildenwall, H., Peterson, S., KadduMulindwa, D., Kalyesubula, I. \&Tumwine, J. K. (2008) Bacterial aetiology and outcome in children with severe pneumonia in Uganda. Annals of Tropical Paediatrics: International Child Health 28: 253-260.

[34] Nelson, P. W., Gilchrist, M. A., Coombs, D., Hyman, J. M., \& Perelson, A. S. (2004). An age-structured model of HIV infection that allows for variations in the death rate of productively infected cells; Math. Bioscience 1: 267-288. Doi: 10.3934/mbe.2004.1.267.

[35] Nuorti, P., Butler, J. C., Crutcher, J., Guevara, R., Welch, D., Holder, P., et al. (1998). An outbreak of multidrugresistantant pneumococcal pneumonia and bacteremia among unvaccinated nursing rome residents. N. Engl. J. Med. 338: 1861-1868. Doi: 10.1056/NEJM199806253382601.

[36] O’Brien, K. L., Wolfson, L. J., Watt, J. P., Henkle, E., Deloria-Knoll, M., McCall, N., Lee, E., Mulholland, K., Levine, O. S. \& Cherian, T. (2009) Burden of disease caused by Streptococcus pneumoniae in children younger than 5 years: global estimates. Lancet 374: 893-902.

[37] Prina, E., Ranzani, O. T., \& Torres, A. (2015b). Communityacquired pneumonia; Lancet 386: 1097-1108. doi: 10.1016/S0140-6736(15)60733-4.

[38] Schrag, S. J., Peña, C., Fernández, J., Sánchez, J., Gómez, V., Pérez, E., et al. (2001). Effect of short-course, high-dose amoxicillin therapy on resistant pneumococcal carriage: a randomized trial. JAMA 286: 49-56. Doi: 10.1001/jama.286.1.49. Available online at: http://jamanetwork.com/journals/jama/fullarticle/193977.

[39] Shrestha, S., Foxman, B., Dawid, S., Aiello, A. E., Davis, B. M., Berus, J., et al. (2013). Time and dose-dependent risk of pneumococcal pneumonia following influenza: a model for within-host interaction between influenza and Streptococcus pneumoniae. Interf. Focus 10: 20130233. doi: 10.1098/rsif.2013.0233.

[40] Smith, A. M., Adler, F. R., Ribeiro, R. M., Gutenkunst, R. N., McAuley, J. L., McCullers, J. A., et al. (2013). Kinetics of coinfection with influenza A virus and Streptococcus pneumoniae. PloSPathog. 9:e1003238. Doi: 10.1371/journal.ppat.1003238.

[41] Smith, A. M., McCullers, J. A., \& Adler, F. R. (2011). Mathematical model of a three-stage innate immune response to a pneumococcal lung infection; J. Theoritical Biology 276: 106-116. doi: 10.1016/j.jtbi.2011.01.052.

[42] Van den Driessche \& Watmough J. "Reproduction numbers and sub-threshold endemic equilibria for compartmental models of disease transmission"; Mathematics Biosciences, 180 (2): $29-48$.

[43] Wang W. \& Ruan S. (2004), Simulating the SARS outbreak in Beijing with limited data, J. Theoretical Biology227: 369-379.

[44] Xu, R. (2012) Global stability of a delayed epidemic model with latent period and vaccination strategy Appl. Math. Model, 36: 5293-5300.

[45] Zhang X, \& Liu X. N. (2008) Backward bifurcation of an epidemic model with saturated treatment function. Journal of Mathematical Analysis and Applications; 348 (1): 433-443.

[46] Zhou L, \& Fan M. (2012) Dynamics of an SIR epidemic model with limited medical resources revisited. Nonlinear Analysis: Real World Applications; 13 (1): 312-324.

[47] Omame, A., Okuonghae, D., Umana, R. A., Inyama, S. C., (2019) Analysis of a co-infection model for HPV-TB, Applied Mathematical https://doi.org/10.1016/j.apm.2019.08.012. 